\title{
CAIS
}

\section{Some Characterizations of $h$-Regular $\Gamma$-Hemiring in terms of Cubic $h$-Ideals}

\author{
Debabrata Mandal ${ }^{1 *}$
}

\begin{abstract}
The aim of this paper is to study $h$-hemiregular and $h$-intra-hemiregular $\Gamma$-hemiring using the combined concept of cubic set and $h$-ideals. We have defined two types of compositions of cubic sets and used these to obtain some characterizations of $h$-hemiregular and $h$-intra-hemiregular $\Gamma$-hemiring.
\end{abstract}

Keywords: $\Gamma$-hemiring, Cubic (h-, h-bi-, h-quasi-) ideal, h-intra-hemiregular, h-hemiregular. 2010 AMS: Primary 08A72, Secondary 16Y99

${ }^{1}$ Department of Mathematics, Raja Peary Mohan College, Uttarpara, Hooghly, India -712258. ORCID: 0000-0001-8605-7865

*Corresponding author: dmandaljumath@gmail.com

Received: 1 June 2020, Accepted: 17 December 2020, Available online: 22 December 2020

\section{Introduction}

Semirings [3], a common generalization of rings and distributive lattices, arise naturally in graph theory, automata theory, mathematical modelling, functional analysis etc.. We know that ideals of ring are a very important tool to describe the structure theory and it is useful in various purposes. If we try to obtain similar results in case of semiring, we find that there are some limitations. To make the connection between the ring ideals and semiring ideals Henriksen [4] defined a special kind of ideals, called $k$-ideal. Iizuka [5] extended the results to a similar kind of ideals called $h$-ideals. As a continuation of this, La Torre [10] studied $h$-ideals and $k$-ideals in hemirings and tried to investigate the gap between the ring ideals and semiring ideals.

In 1965, Zadeh [16] proposed the theory of fuzzy sets. After that we have seen that it is a very useful mathematical tool for describing the vague or complex or illdefined systems. Rosenfeld [15] used the concept to study of fuzzy algebraic structure. Since then many researchers have developed these ideas. Jun et al [6] applied the concept to initiate the study of fuzzy $h$-ideals in hemiring. Sardar et al [13, 14], Ma et al [11] extended some of these results in more general setting of hemiring i.e. $\Gamma$-hemiring. Jun et al [7, 8] initiated the study of cubic subgroups and cubic sets. Khan et al [9] applied this in case of cubic $h$-ideals of hemirings. Chinnadurai $[1,2]$ used this notion to study cubic bi-ideals and cubic lateral ideals in near-ring and ternary near-ring respectively.

As a continuation of this, the main aim of this paper is to study $h$-hemiregularity and $h$-intra-hemiregularity criterion of $\Gamma$-hemiring using cubic $h$-ideals, cubic $h$-bi-ideals and cubic $h$-quasi-ideals.

\section{Primary Ideas}

We know that a hemiring is a nonempty set $S$ on which operations addition and multiplication have been defined such that $(S,+)$ is a commutative monoid with identity $0,(S, \cdot)$ is a semigroup and multiplication distributes over addition from either side. In addition to that, $0 \cdot s=0=s \cdot 0$ for all $s \in S$. As an extension of this $\Gamma$-hemiring can be defined as follows:

For two additive commutative semigroups with zero, $S$ and $\Gamma$, there exists a mapping $S \times \Gamma \times S \rightarrow S((a, \alpha, b) \mapsto a \alpha b)$ which satisfy the following conditions: 
i) $(a+b) \alpha c=a \alpha c+b \alpha c$,

ii) $a \alpha(b+c)=a \alpha b+a \alpha c$,

iii) $a(\alpha+\beta) b=a \alpha b+a \beta b$,

iv) $a \alpha(b \beta c)=(a \alpha b) \beta c$,

v) $0_{S} \alpha a=0_{S}=a \alpha 0_{S}$,

vi) $a 0_{\Gamma} b=0_{S}=b 0_{\Gamma} a$

for all $a, b, c \in S$ and for all $\alpha, \beta \in \Gamma$.

We now summon up the definitions of several types of ideals.

A subset $I$ of a $\Gamma$-hemiring $S$ is called a left(resp. right) ideal of $S$ if $I$ is closed under addition and $S \Gamma I \subseteq I$ (resp. $I \Gamma S \subseteq I$ ).

A subset $Q$ of a $\Gamma$-hemiring $S$ is called a quasi-ideal of $S$ if $Q$ is closed under addition and $S \Gamma Q \cap Q \Gamma S \subseteq Q$.

A subset $B$ of a $\Gamma$-hemiring $S$ is called a bi-ideal if $B$ is closed under addition and $B \Gamma S \Gamma B \subseteq B$.

A left ideal $H$ of $S$ is called a left $h$-ideal if $x, z \in S, a, b \in H$ and $x+a+z=b+z$ implies $x \in H$. A right $h$-ideal is defined analoguesly.

We now remind the definition of cubic set and characteristic cubic set. For a non-empty set $X$, a cubic set $C$ in $X$ is a structure $C=<\widetilde{\mu}, f>$ where $\widetilde{\mu}=\left[\mu^{-}, \mu^{+}\right]$is an interval valued fuzzy set and $f$ is a fuzzy set in $X$. For any non-empty set $G$ of a set $X$, the characteristic cubic set of $G$ is defined to be the structure $\chi_{G}(x)=<x, \widetilde{\zeta}_{\chi_{G}}(x), \eta_{\chi_{G}}(x): x \in X>$ where

$$
\widetilde{\zeta}_{\chi_{G}}(x)=\left\{\begin{array}{l}
{[1,1] \approx \widetilde{1} \text { if } x \in G} \\
{[0,0] \approx \widetilde{0} \text { otherwise. }}
\end{array}\right.
$$

and

$$
\eta_{\chi_{G}}(x)= \begin{cases}0 & \text { if } x \in G \\ 1 & \text { otherwise }\end{cases}
$$

\section{Cubic $h$-Ideals}

In this section, we recall some definitions and results from [12] which will be used to develop the main portion of the paper.

Definition 3.1. Let $\langle\widetilde{\mu}, f>$ be a non empty cubic subset of a $\Gamma$-hemiring $S$. Then $\langle\widetilde{\mu}, f>$ is called a cubic left ideal [respectively, cubic right ideal] of $S$ if

(i) $\widetilde{\mu}(x+y) \supseteq \cap\{\widetilde{\mu}(x), \widetilde{\mu}(y)\}, f(x+y) \leq \max \{f(x), f(y)\}$ and

(ii) $\widetilde{\mu}(x \gamma y) \supseteq \widetilde{\mu}(y), f(x \gamma y) \leq f(y)$ [respectively, $\widetilde{\mu}(x \gamma y) \supseteq \widetilde{\mu}(x), f(x \gamma y) \leq f(x)]$.

for all $x, y \in S, \gamma \in \Gamma$.

Note: For cubic left or right ideal $<\widetilde{\mu}, f>$ of a $\Gamma$-hemiring $S, \widetilde{\mu}(0) \supseteq \widetilde{\mu}(x)$ and $f(0) \leq f(x)$ for all $x \in S$.

Definition 3.2. A cubic left ideal $<\widetilde{\mu}, f>$ of $a$-hemiring $S$ is called a cubic left h-ideal iffor all $a, b, x, z \in S, x+a+z=b+z$ then $\widetilde{\mu}(x) \supseteq \cap\{\widetilde{\mu}(a), \widetilde{\mu}(b)\}$ and $f(x) \leq \max \{f(a), f(b)\}$.

Definition 3.3. Let $A=<\widetilde{\mu}, f>$ and $B=<\widetilde{\boldsymbol{\theta}}, g>$ be two cubic sets of $a \Gamma-$ hemiring $S$. Define intersection of $A$ and $B$ by

$$
A \cap B=<\widetilde{\mu}, f>\cap<\widetilde{\theta}, g>=<\widetilde{\mu} \cap \widetilde{\theta}, f \cup g>.
$$

Definition 3.4. Let $A=<\widetilde{\mu}, f>$ and $B=<\widetilde{\theta}, g>$ be two cubic sets of $a \Gamma$-hemiring $S$. Define composition of $A$ and $B$ by

$$
A \Gamma_{c h} B=<\widetilde{\mu}, f>\Gamma_{c h}<\widetilde{\theta}, g>=<\widetilde{\mu} \Gamma_{c h} \widetilde{\theta}, f \Gamma_{c h} g>
$$

where

$$
\begin{aligned}
\widetilde{\mu} \Gamma_{c h} \widetilde{\theta}(x) & =\underset{x}{\cup}\left[\cap\left\{\widetilde{\mu}\left(a_{1}\right), \widetilde{\mu}\left(a_{2}\right), \widetilde{\theta}\left(b_{1}\right), \widetilde{\theta}\left(b_{2}\right)\right\}\right] \\
& =\underset{0}{ } \tilde{0}, \text { if } x b_{1}+z=a_{2} \delta b_{2}+z \\
&
\end{aligned}
$$


and

$$
\begin{aligned}
f \Gamma_{\text {ch }} g(x)= & \inf \left\{\max \left\{f\left(a_{1}\right), f\left(a_{2}\right), g\left(b_{1}\right), g\left(b_{2}\right)\right\}\right\} \\
& x+a_{1} \gamma b_{1}+z=a_{2} \delta b_{2}+z \\
= & 1, \text { if } x \text { cannot be expressed as above }
\end{aligned}
$$

for $x, z, a_{1}, a_{2}, b_{1}, b_{2} \in S$ and $\gamma, \delta \in \Gamma$.

Definition 3.5. Let $A=<\widetilde{\mu}, f>$ and $B=<\widetilde{\theta}, g>$ be two cubic sets of $a \Gamma-$ hemiring $S$. Define generalized composition of $A$ and $B$ by

$$
A o_{c h} B=<\widetilde{\mu}, f>o_{c h}<\widetilde{\boldsymbol{\theta}}, g>=<\widetilde{\mu} o_{c h} \widetilde{\boldsymbol{\theta}}, f o_{c h} g>
$$

where

$$
\begin{aligned}
\widetilde{\mu} o_{c h} \widetilde{\theta}(x)= & \cup\left[\cap\left\{\cap\left\{\widetilde{\mu}\left(a_{i}\right), \widetilde{\mu}\left(c_{i}\right), \widetilde{\theta}\left(b_{i}\right), \widetilde{\theta}\left(d_{i}\right)\right\}\right\}\right] \\
& x+\sum_{i=1}^{n} a_{i} \gamma_{i} b_{i}+z=\sum_{i=1}^{n} c_{i} \delta_{i} d_{i}+z \\
= & \widetilde{0}, \text { if } x \text { cannot be expressed as above }
\end{aligned}
$$

and

$$
\begin{aligned}
f o_{c h} g(x)= & \inf \left[\max _{i}\left\{\max \left\{f\left(a_{i}\right), f\left(c_{i}\right), g\left(b_{i}\right), g\left(d_{i}\right)\right\}\right\}\right] \\
& x+\sum_{i=1}^{n} a_{i} \gamma_{i} b_{i}+z=\sum_{i=1}^{n} c_{i} \delta_{i} d_{i}+z \\
= & 1, \text { if x cannot be expressed as above }
\end{aligned}
$$

where $x, z, a_{i}, b_{i}, c_{i}, d_{i} \in S$ and $\gamma_{i}, \delta_{i} \in \Gamma$, for $i \in\{1, \ldots, n\}$.

Lemma 3.6. Let $A=<\widetilde{\mu}_{1}, f>, B=<\widetilde{\mu}_{2}, g>$ be two cubic h-ideal of a $\Gamma$-hemiring $S$. Then $A \Gamma_{c h} B \subseteq A o_{c h} B \subseteq A \cap B \subseteq$ $A($ and $B)$.

Definition 3.7. A cubic subset $<\widetilde{\mu}, f>$ of a $\Gamma$-hemiring $S$ is called cubic h-bi-ideal iffor all $x, y, z, a, b \in S$ and $\alpha, \beta \in \Gamma$ we have

i) $\widetilde{\mu}(x+y) \supseteq \cap\{\widetilde{\mu}(x), \widetilde{\mu}(y)\}, f(x+y) \leq \max \{f(x), f(y)\}$

ii) $\widetilde{\mu}(x \alpha y) \supseteq \cap\{\widetilde{\mu}(x), \widetilde{\mu}(y)\}, f(x \alpha y) \leq \max \{f(x), f(y)\}$

iii) $\widetilde{\mu}(x \alpha y \beta z) \supseteq \cap\{\widetilde{\mu}(x), \widetilde{\mu}(z)\}, f(x \alpha y \beta z) \leq \max \{f(x), f(z)\}$

iv) If $x+a+z=b+z$ then $\widetilde{\mu}(x) \supseteq \cap\{\widetilde{\mu}(a), \widetilde{\mu}(b)\}, f(x) \leq \max \{f(a), f(b)\}$

Definition 3.8. A cubic subset $<\widetilde{\mu}, f>$ of $a \Gamma$-hemiring $S$ is called cubic h-quasi-ideal if for all $x, y, z, a, b \in S$ we have

i) $\widetilde{\mu}(x+y) \supseteq \cap\{\widetilde{\mu}(x), \widetilde{\mu}(y)\}, f(x+y) \leq \max \{f(x), f(y)\}$

ii) $\left(\widetilde{\mu} o_{c h} \widetilde{\zeta}_{\chi_{S}}\right) \cap\left(\widetilde{\zeta}_{\chi_{S} o_{c h}} \widetilde{\mu}\right) \subseteq \widetilde{\mu},\left(f o_{c h} \eta_{\chi_{S}}\right) \cup\left(\eta_{\chi_{S}} o_{c h} f\right) \supseteq f$,

iii) If $x+a+z=b+z$ then $\widetilde{\mu}(x) \supseteq \cap\{\widetilde{\mu}(a), \widetilde{\mu}(b)\}, f(x) \leq \max \{f(a), f(b)\}$

Lemma 3.9. Any cubic h-quasi-ideal of $S$ is a cubic h-bi-ideal of $S$.

\section{Cubic $\boldsymbol{h}$-Hemiregularity and Cubic $\boldsymbol{h}$-Intra-Hemiregularity}

In this section, we study the concept of $h$-hemiregularity and $h$-intra-hemiregularity in $\Gamma$-hemiring by using cubic $h$-ideal, cubic $h$-bi-ideal and cubic $h$-quasi-ideal.

Definition 4.1. [11] A $\Gamma$-hemiring $S$ is said to be h-hemiregular if for each $x \in S$, there exist $a, b \in S$ and $\alpha, \beta, \gamma, \delta \in \Gamma$ such that $x+x \alpha a \beta x+z=x \gamma b \delta x+z$.

We now try to find some characterizations of $h$-hemiregular $\Gamma$-hemiring in terms of cubic $h$-ideals. 
Theorem 4.2. Let $S$ be an h-hemiregular $\Gamma$-hemiring. Then for any cubic right h-ideal $A=<\widetilde{\mu}, f>$ and any cubic left h-ideal $B=<\widetilde{v}, g>$ of $S$, we have $A \Gamma_{c h} B=A \cap B$.

Proof. Let $S$ be an $h$-hemiregular $\Gamma$-hemiring. By Lemma 3.6, we have $A \Gamma_{c h} B \subseteq A \cap B$.

Since $S$ is $h$-hemiregular, for any $a \in S$, there exist $z, x_{1}, x_{2} \in S$ and $\alpha_{1}, \beta_{1}, \alpha_{2}, \beta_{2} \in \Gamma$ such that $a+a \alpha_{1} x_{1} \beta_{1} a+z=a \alpha_{2} x_{2} \beta_{2} a+z$. Now for any $e, b, c, d \in S$ and $\gamma, \delta \in \Gamma$, the general expression of $a$ as $a+e \gamma b+z=c \delta d+z$ implies that

$$
\begin{aligned}
& \left(\widetilde{\mu} \Gamma_{c h} \widetilde{v}\right)(a)=\underset{a+e \gamma b+z=c \delta d+z}{\cup\{\cap\{\widetilde{\mu}(e), \widetilde{\mu}(c), \widetilde{v}(b), \widetilde{v}(d)\}\}} \\
& \supseteq \cap\left\{\widetilde{\mu}\left(a \alpha_{1} x_{1}\right), \widetilde{\mu}\left(a \alpha_{2} x_{2}\right), \widetilde{v}(a)\right\} \\
& a+a \alpha_{1} x_{1} \beta_{1} a+z=a \alpha_{2} x_{2} \beta_{2} a+z \\
& \supseteq \cap\{\widetilde{\mu}(a), \widetilde{\mu}(a), \widetilde{v}(a)\} \\
& =\cap\{\widetilde{\mu}(a), \widetilde{v}(a)\}=(\widetilde{\mu} \cap \widetilde{v})(a) . \\
& \left.\left(f \Gamma_{c h} g\right)(a)=\inf _{a+e \gamma b+z=c \delta d+z}\{f(e), f(c), g(b), g(d)\}\right\} \\
& \leq \max \left\{f\left(a \alpha_{1} x_{1}\right), f\left(a \alpha_{2} x_{2}\right), g(a)\right\} \\
& a+a \alpha_{1} x_{1} \beta_{1} a+z=a \alpha_{2} x_{2} \beta_{2} a+z \\
& \leq \max \{f(a), f(a), g(a)\} \\
& =\max \{f(a), g(a)\}=(f \cup g)(a) .
\end{aligned}
$$

Therefore $(A \cap B) \subseteq\left(A \Gamma_{c h} B\right)$.

Hence $A \Gamma_{c h} B=A \cap B$.

Corollary 4.3. If $S$ be a h-hemiregular $\Gamma$-hemiring, then for any cubic right $h$-ideal $A=<\widetilde{\mu}, f>$ and any cubic left h-ideal $B=<\widetilde{v}, g>$ of $S$ we have $A o_{c h} B=A \cap B$.

Theorem 4.4. Let $S$ be a h-hemiregular $\Gamma$-hemiring. Then

(i) $A \subseteq A o_{c h} \chi_{S} o_{c h} A$ for every cubic h-bi-ideal $A=<\widetilde{\mu}, f>$ of $S$.

(ii) $A \subseteq A o_{c h} \chi_{S} o_{c h} A$ for every cubic h-quasi-ideal $A=<\widetilde{\mu}, f>$ of $S$.

Proof. Suppose that $A=<\widetilde{\mu}, f>$ be any cubic h-bi-ideal of $S$ and $x$ be any element of $S$. Since $S$ is $h$-hemiregular there exist $a, b, z \in S$ and $\alpha, \beta, \gamma, \delta \in \Gamma$ such that $x+x \alpha a \beta x+z=x \gamma b \delta x+z$.

Now for any general expression of $x$ as $x+\sum_{i=1}^{n} a_{i} \gamma_{i} b_{i}+z=\sum_{i=1}^{n} c_{i} \delta_{i} d_{i}+z$, where $a_{i}, b_{i}, c_{i}, d_{i} \in S$ and $\gamma_{i}, \delta_{i} \in \Gamma$, we have

$$
\left(\widetilde{\mu} o_{c h} \widetilde{\zeta}_{\chi_{S}} o_{c h} \widetilde{\mu}\right)(x)
$$

$$
\begin{gathered}
=\cup\left(\cap\left\{\left(\widetilde{\mu} o_{c h} \widetilde{\zeta}_{\chi_{S}}\right)\left(a_{i}\right),\left(\widetilde{\mu} o_{c h} \widetilde{\zeta}_{\chi_{S}}\right)\left(c_{i}\right), \widetilde{\mu}\left(b_{i}\right), \widetilde{\mu}\left(d_{i}\right)\right\}\right) \\
x+\sum_{i=1}^{n} a_{i} \gamma_{i} b_{i}+z=\sum_{i=1}^{n} c_{i} \delta_{i} d_{i}+z
\end{gathered}
$$

$\supseteq \cap\left\{\left(\widetilde{\mu} o_{c h} \widetilde{\zeta}_{\chi_{S}}\right)(x \alpha a),\left(\widetilde{\mu} o_{c h} \widetilde{\zeta}_{\chi_{S}}\right)(x \gamma b), \widetilde{\mu}(x)\right\}$

$$
x+x \alpha a \beta x+z=x \gamma b \delta x+z
$$

$$
=\cap\left\{\quad \cup\left(\cap\left\{\left(\widetilde{\mu}\left(a_{i}\right), \widetilde{\mu}\left(c_{i}\right)\right)\right\}\right) \quad, \quad \cup\left(\cap\left\{\left(\widetilde{\mu}\left(a_{i}\right), \widetilde{\mu}\left(c_{i}\right)\right)\right\}\right) \quad,\right.
$$

$$
x \alpha a+\sum_{i=1}^{n} a_{i} \gamma_{i} b_{i}+z=\sum_{i=1}^{n} c_{i} \delta_{i} d_{i}+z x \gamma b+\sum_{i=1}^{n} a_{i} \gamma_{i} b_{i}+z=\sum_{i=1}^{n} c_{i} \delta_{i} d_{i}+z
$$

$\widetilde{\mu}(x)\}$

$$
\begin{aligned}
\supseteq & \cap\{\widetilde{\mu}(x), \widetilde{\mu}(x), \widetilde{\mu}(x)\}(\text { since } x \alpha a+x \alpha a \beta x \alpha a+z \alpha a=x \gamma b \delta x \alpha a+z \alpha a \text { and } x \gamma b+x \alpha a \beta x \gamma b+z \gamma b=x \gamma b \delta x \gamma b+z \gamma b) . \\
= & \widetilde{\mu}(x) . \\
& \quad\left(f o_{c h} \eta_{\chi_{S}} o_{c h} f\right)(x) \\
= & \inf \left(\max \left\{\left(f o_{c h} \eta_{\chi_{S}}\right)\left(a_{i}\right),\left(f o_{c h} \eta_{\chi_{S}}\right)\left(c_{i}\right), f\left(b_{i}\right), f\left(d_{i}\right)\right\}\right) \\
\quad & \quad x+\sum_{i=1}^{n} a_{i} \gamma_{i} b_{i}+z=\sum_{i=1}^{n} c_{i} \delta_{i} d_{i}+z
\end{aligned}
$$




$$
\begin{aligned}
& \leq \max \left\{\left(f o_{c h} \eta_{\chi_{S}}\right)(x \alpha a),\left(f o_{c h} \eta_{\chi_{S}}\right)(x \gamma b), f(x)\right\} \\
& =\max \left\{\quad \inf \left(\max \left\{\left(f\left(a_{i}\right), f\left(c_{i}\right)\right)\right\}\right) \quad, \quad \inf \left(\max \left\{\left(f\left(a_{i}\right), f\left(c_{i}\right)\right)\right\}\right), f(x)\right\} \\
& \quad x \alpha a+\sum_{i=1}^{n} a_{i} \gamma_{i} b_{i}+z=\sum_{i=1}^{n} c_{i} \delta_{i} d_{i}+z x \gamma b+\sum_{i=1}^{n} a_{i} \gamma_{i} b_{i}+z=\sum_{i=1}^{n} c_{i} \delta_{i} d_{i}+z \\
& \leq \max \{f(x), f(x), f(x)\}(\text { since } x \alpha a+x \alpha a \beta x \alpha a+z \alpha a=x \gamma b \delta x \alpha a+z \alpha a \text { and } x \gamma b+x \alpha a \beta x \gamma b+z \gamma b=x \gamma b \delta x \gamma b+z \gamma b) . \\
& =f(x) .
\end{aligned}
$$

This implies that $A \subseteq A o_{c h} \chi_{S} o_{c h} A$.

(i) $\Rightarrow$ (ii) By Lemma 3.9 "Any cubic $h$-quasi-ideal of $S$ is a cubic $h$-bi-ideal of $S$ ". Thus, if the proof of (ii) is made, it is straightforward to see that (i) is true by Lemma 3.9.

Theorem 4.5. Let $S$ be a h-hemiregular $\Gamma$-hemiring. Then

(i) $A \cap B \subseteq A o_{c h} B o_{c h} A$ for every cubic h-bi-ideal $A=<\widetilde{\mu}, f>$ and every cubic $h$-ideal $B=<\widetilde{v}, g>$ of $S$.

(ii) $A \cap B \subseteq A o_{c h} B o_{c h} A$ for every cubic h-quasi-ideal $A=<\widetilde{\mu}, f>$ and every cubic $h$-ideal $B=<\widetilde{v}, g>$ of $S$.

Proof. Suppose $S$ is a $h$-hemiregular $\Gamma$-hemiring and $A=<\widetilde{\mu}, f>, B=<\widetilde{v}, g>$ be any cubic $h$-bi-ideal and cubich-ideal of $S$, respectively and $x$ be any element of $S$. Since $S$ is $h$-hemiregular, there exist $a, b, z \in S$ and $\alpha, \beta, \gamma, \delta \in \Gamma$ such that $x+x \alpha a \beta x+z=x \gamma b \delta x+z$.

Now for any general expression of $x$ as $x+\sum_{i=1}^{n} a_{i} \gamma_{i} b_{i}+z=\sum_{i=1}^{n} c_{i} \delta_{i} d_{i}+z$, where $a_{i}, b_{i}, c_{i}, d_{i} \in S$ and $\gamma_{i}, \delta_{i} \in \Gamma$, we have

$$
\begin{aligned}
& \left(\widetilde{\mu} o_{c h} \widetilde{v} o_{c h} \widetilde{\mu}\right)(x) \\
& =\cup\left(\cap\left\{\left(\widetilde{\mu} o_{c h} \widetilde{v}\right)\left(a_{i}\right),\left(\widetilde{\mu} o_{c h} \widetilde{v}\right)\left(c_{i}\right), \widetilde{\mu}\left(b_{i}\right), \widetilde{\mu}\left(d_{i}\right)\right\}\right) \\
& x+\sum_{i=1}^{n} a_{i} \gamma_{i} b_{i}+z=\sum_{i=1}^{n} c_{i} \delta_{i} d_{i}+z \\
& \supseteq \cap\left\{\left(\widetilde{\mu} o_{c h} \widetilde{v}\right)(x \alpha a),\left(\widetilde{\mu} o_{c h} \widetilde{v}\right)(x \gamma b), \widetilde{\mu}(x)\right\} \\
& x+x \alpha a \beta x+z=x \gamma b \delta x+z \\
& =\cap\left\{\cup\left(\cap\left\{\left(\widetilde{\mu}\left(a_{i}\right), \widetilde{\mu}\left(c_{i}\right), \widetilde{v}\left(b_{i}\right), \widetilde{v}\left(d_{i}\right)\right)\right\}\right), \cup\left(\cap\left\{\left(\widetilde{\mu}\left(a_{i}\right), \widetilde{\mu}\left(c_{i}\right), \widetilde{v}\left(b_{i}\right), \widetilde{v}\left(d_{i}\right)\right)\right\}\right),\right. \\
& x \alpha a+\sum_{i=1}^{n} a_{i} \gamma_{i} b_{i}+z=\sum_{i=1}^{n} c_{i} \delta_{i} d_{i}+z \quad x \gamma b+\sum_{i=1}^{n} a_{i} \gamma_{i} b_{i}+z=\sum_{i=1}^{n} c_{i} \delta_{i} d_{i}+z
\end{aligned}
$$

$\widetilde{\mu}(x)\}$

$\supseteq \cap\{\cap\{\widetilde{\mu}(x), \widetilde{v}(a \beta x \alpha a), \widetilde{v}(b \delta x \alpha a)\}, \cap\{\widetilde{\mu}(x), \widetilde{v}(a \beta x \gamma b), \widetilde{v}(b \delta x \gamma b)\}, \widetilde{\mu}(x)\}$

(since $x \alpha a+x \alpha a \beta x \alpha a+z \alpha a=x \gamma b \delta x \alpha a+z \alpha a$ and $x \gamma b+x \alpha a \beta x \gamma b+z \gamma b=x \gamma b \delta x \gamma b+z \gamma b)$

$\supseteq \cap\{\widetilde{\mu}(x), \widetilde{v}(x)\}=(\widetilde{\mu} \cap \widetilde{v})(x)$.

$$
\left(f o_{c h} g o_{c h} f\right)(x)
$$

$$
\begin{gathered}
=\inf \left(\max \left\{\left(f o_{c h} g\right)\left(a_{i}\right),\left(f o_{c h} g\right)\left(c_{i}\right), f\left(b_{i}\right), g\left(d_{i}\right)\right\}\right) \\
x+\sum_{i=1}^{n} a_{i} \gamma_{i} b_{i}+z=\sum_{i=1}^{n} c_{i} \delta_{i} d_{i}+z
\end{gathered}
$$

$\leq \max \left\{\left(f o_{c h} g\right)(x \alpha a),\left(f o_{c h} g\right)(x \gamma b), f(x)\right\}$

$$
x+x \alpha a \beta x+z=x \gamma b \delta x+z
$$

$=\max \left\{\inf \left(\max \left\{\left(f\left(a_{i}\right), f\left(c_{i}\right), g\left(b_{i}\right), g\left(d_{i}\right)\right)\right\}\right), \inf \left(\max \left\{\left(f\left(a_{i}\right), f\left(c_{i}\right), g\left(b_{i}\right), g\left(d_{i}\right)\right)\right\}\right)\right.$,

$$
x \alpha a+\sum_{i=1}^{n} a_{i} \gamma_{i} b_{i}+z=\sum_{i=1}^{n} c_{i} \delta_{i} d_{i}+z \quad x \gamma b+\sum_{i=1}^{n} a_{i} \gamma_{i} b_{i}+z=\sum_{i=1}^{n} c_{i} \delta_{i} d_{i}+z
$$

$f(x)\}$

$\leq \max \{\max \{f(x), g(a \beta x \alpha a), g(b \delta x \alpha a)\}, \cap\{f(x), g(a \beta x \gamma b), g(b \delta x \gamma b)\}, f(x)\}$

(since $x \alpha a+x \alpha a \beta x \alpha a+z \alpha a=x \gamma b \delta x \alpha a+z \alpha a$ and $x \gamma b+x \alpha a \beta x \gamma b+z \gamma b=x \gamma b \delta x \gamma b+z \gamma b)$

$\supseteq \max \{f(x), g(x)\}=(f \cup g)(x)$.

(i) $\Rightarrow$ (ii) This is straightforward using the Lemma 3.9.

Definition 4.6. [13]A $\Gamma$-hemiring $S$ is said to be h-intra-hemiregular if for each $x \in S$, there exist $z, a_{i}, a_{i}^{\prime}, b_{i}, b_{i}^{\prime} \in S$, and $\alpha_{i}, \beta_{i}, \gamma_{i}, \delta_{i}, \eta \in \Gamma, i \in \mathbf{N}$, such that $x+\sum_{i=1}^{n} a_{i} \alpha_{i} x \eta x \beta_{i} a_{i}^{\prime}+z=\sum_{i=1}^{n} b_{i} \gamma_{i} x \eta x \delta b_{i}^{\prime}+z$.

We now try to find a characterization of $h$-intrahemiregular $\Gamma$-hemiring in terms of cubic $h$-ideals. 
Theorem 4.7. Let $S$ be a h-intra-regular $\Gamma$-hemiring. Then $A \cap B \subseteq A o_{c h} B$ for every cubic left h-ideal $A=<\tilde{\mu}, f>$ and every cubic right h-ideal $A=<\widetilde{v}, g>$ of $S$.

Proof. Suppose $S$ is $h$-intra-hemiregular. Let $A=<\widetilde{\mu}, f>$ and $A=<\widetilde{v}, g>$ be any cubic left $h$-ideal and cubic right $h$-ideal of $S$ respectively. Now let $x \in S$. Then by hypothesis there exist $z, a_{i}, a_{i}^{\prime}, b_{i}, b_{i}^{\prime} \in \mathrm{S}$, and $\alpha_{i}, \beta_{i}, \gamma_{i}, \delta_{i}, \eta \in \Gamma, \mathrm{i} \in \mathbf{N}$, the set of natural numbers, such that $x+\sum_{i=1}^{n} a_{i} \alpha_{i} x \eta x \beta_{i} a_{i}^{\prime}+z=\sum_{i=1}^{n} b_{i} \gamma_{i} x \eta x \delta b_{i}^{\prime}+z$. Now for any general expression of $x$ as $x+\sum_{i=1}^{n} a_{i} \gamma_{i} b_{i}+z=\sum_{i=1}^{n} c_{i} \delta_{i} d_{i}+z$, where $a_{i}, b_{i}, c_{i}, d_{i} \in S$ and $\gamma_{i}, \delta_{i} \in \Gamma$, we have

$$
\begin{aligned}
\left(\widetilde{\mu} o_{c h} \widetilde{v}\right)(x)= & \cup\left[\bigcap_{i}\left\{\cap\left\{\widetilde{\mu}\left(a_{i}\right), \widetilde{\mu}\left(c_{i}\right), \widetilde{v}\left(b_{i}\right), \widetilde{v}\left(d_{i}\right)\right\}\right\}\right] \\
& x+\sum_{i=1}^{n} a_{i} \gamma_{i} b_{i}+z=\sum_{i=1}^{n} c_{i} \delta_{i} d_{i}+z \\
\supseteq & \bigcap_{i}\left[\cap\left\{\widetilde{\mu}\left(a_{i} \alpha_{i} x\right), \widetilde{\mu}\left(b_{i} \gamma_{i} x\right), \widetilde{v}\left(x \beta_{i} a_{i}^{\prime}\right), \widetilde{v}\left(x \delta_{i} b_{i}^{\prime}\right)\right\}\right] \\
& x+\sum_{i=1}^{n} a_{i} \alpha_{i} x \eta x \beta_{i} a_{i}^{\prime}+z=\sum_{i=1}^{n} b_{i} \gamma_{i} x \eta x \delta b_{i}^{\prime}+z \\
\supseteq & \cap\{\widetilde{\mu}(x), \widetilde{v}(x)\}=(\widetilde{\mu} \cap \widetilde{v})(x) . \\
\left(f o_{c h} g\right)(x)= & \inf \left[\max _{i}\left\{\max \left\{f\left(a_{i}\right), f\left(c_{i}\right), g\left(b_{i}\right), g\left(d_{i}\right)\right\}\right\}\right] \\
& x+\sum_{i=1}^{n} a_{i} \gamma_{i} b_{i}+z=\sum_{i=1}^{n} c_{i} \delta_{i} d_{i}+z \\
\leq & \max _{i}\left[\max \left\{f\left(a_{i} \alpha_{i} x\right), f\left(b_{i} \gamma_{i} x\right), g\left(x \beta_{i} a_{i}^{\prime}\right), g\left(x \delta_{i} b_{i}^{\prime}\right)\right\}\right] \\
& \quad x+\sum_{i=1}^{n} a_{i} \alpha_{i} x \eta x \beta_{i} a_{i}^{\prime}+z=\sum_{i=1}^{n} b_{i} \gamma_{i} x \eta x \delta b_{i}^{\prime}+z \\
\leq & \max \{f(x), g(x)\}=(f \cup g)(x) .
\end{aligned}
$$

Hence the proof is completed.

We now combine the concepts of $h$-hemiregularity and $h$-intra-hemiregularity of a $\Gamma$-hemiring and obtain a characterization.

Theorem 4.8. Let $S$ be both h-hemiregular and h-intra-hemiregular $\Gamma$-hemiring. Then

(i) $A=A o_{c h} A$ for every cubic $h$-bi-ideal $A=<\widetilde{\mu}, f>$ of $S$.

(ii) $A=A o_{c h} A$ for every cubic h-quasi-ideal $A=<\widetilde{\mu}, f>$ of $S$.

Proof. Suppose $S$ be both $h$-hemiregular and $h$-intra-hemiregular $\Gamma$-hemiring. Let $x \in S$ and $A=<\widetilde{\mu}, f>$ be any cubic $h$-bi-ideal of $S$. Since $S$ is both $h$-hemiregular and $h$-intra-hemiregular there exist $z, a_{i}, b_{i}, c_{i}, d_{i} \in S$ and $\alpha_{i}, \beta_{i}, \alpha_{i}^{\prime}, \beta_{i}^{\prime}, \gamma_{i}, \delta_{i}, \gamma_{i}^{\prime}, \delta_{i}^{\prime}, \eta \in \Gamma$, $i \in \mathbf{N}$ such that $x+\sum_{i=1}^{n} x \alpha_{i} a_{i} \alpha_{i}^{\prime} x \eta x \beta_{i}^{\prime} b_{i} \beta_{i} x+z=\sum_{i=1}^{n} x \gamma_{i} c_{i} \gamma_{i}^{\prime} x \eta x \delta_{i}^{\prime} d_{i} \delta_{i} x+z$.

Now for any general expression of $x$ as $x+\sum_{i=1}^{n} a_{i} \gamma_{i} b_{i}+z=\sum_{i=1}^{n} c_{i} \delta_{i} d_{i}+z$, where $a_{i}, b_{i}, c_{i}, d_{i} \in S$ and $\gamma_{i}, \delta_{i} \in \Gamma$, we have

$$
\begin{aligned}
& \left(\widetilde{\mu} o_{c h} \widetilde{\mu}\right)(x) \\
& =\cup\left[\bigcap_{i}\left\{\cap\left\{\widetilde{\mu}\left(a_{i}\right), \widetilde{\mu}\left(c_{i}\right), \widetilde{\mu}\left(b_{i}\right), \widetilde{\mu}\left(d_{i}\right)\right\}\right\}\right] \\
& \quad x+\sum_{i=1}^{n} a_{i} \gamma_{i} b_{i}+z=\sum_{i=1}^{n} c_{i} \delta_{i} d_{i}+z \\
& \supseteq \bigcap_{i}\left[\cap\left\{\widetilde{\mu}\left(x \alpha_{i} a_{i} \alpha_{i}^{\prime} x\right), \widetilde{\mu}\left(x \beta_{i}^{\prime} b_{i} \beta_{i} x\right), \widetilde{\mu}\left(x \gamma_{i} c_{i} \gamma_{i}^{\prime} x\right), \widetilde{\mu}\left(x \delta_{i}^{\prime} d_{i} \delta_{i} x\right)\right\}\right] \\
& \quad x+\sum_{i=1}^{n} x \alpha_{i} a_{i} \alpha_{i}^{\prime} x \eta x \beta_{i}^{\prime} b_{i} \beta_{i} x+z=\sum_{i=1}^{n} x \gamma_{i} c_{i} \gamma_{i}^{\prime} x \eta x \delta_{i}^{\prime} d_{i} \delta_{i} x+z \\
& \supseteq \widetilde{\mu}(x) .
\end{aligned}
$$




$$
\begin{aligned}
& \left(f o_{c h} f\right)(x) \\
= & \inf \left[\max _{i}\left\{\max \left\{f\left(a_{i}\right), f\left(c_{i}\right), f\left(b_{i}\right), f\left(d_{i}\right)\right\}\right\}\right] \\
& x+\sum_{i=1}^{n} a_{i} \gamma_{i} b_{i}+z=\sum_{i=1}^{n} c_{i} \delta_{i} d_{i}+z \\
\leq & \max _{i}\left[\max \left\{f\left(x \alpha_{i} a_{i} \alpha_{i}^{\prime} x\right), f\left(x \beta_{i}^{\prime} b_{i} \beta_{i} x\right), f\left(x \gamma_{i} c_{i} \gamma_{i}^{\prime} x\right), f\left(x \delta_{i}^{\prime} d_{i} \delta_{i} x\right)\right\}\right] \\
& x+\sum_{i=1}^{n} x \alpha_{i} a_{i} \alpha_{i}^{\prime} x \eta x \beta_{i}^{\prime} b_{i} \beta_{i} x+z=\sum_{i=1}^{n} x \gamma_{i} c_{i} \gamma_{i}^{\prime} x \eta x \delta_{i}^{\prime} d_{i} \delta_{i} x+z \\
\leq & f(x) .
\end{aligned}
$$

Now $A o_{c h} A \subseteq A o_{c h} \chi_{S} \subseteq A$. Hence $A o_{c h} A=A$ for every cubic $h$-bi-ideal $A$ of $S$.

(i) $\Rightarrow$ (ii) This is straightforward using the Lemma 3.9 .

\section{Conclusion}

In this paper, I have studied some properties $h$-hemiregular and $h$-intra-hemiregular $\Gamma$-hemiring using the concept of cubic $h$-ideal, cubic $h$-bi-ideal and cubic $h$-quasi-ideal. At the end section, I also acquire some characterizations of $h$-hemiregular and $h$-intra-hemiregular $\Gamma$-hemiring. Interested reader may find some other feature of these types of $\Gamma$-hemiring and extend the obtained result using the concept of neutrosophic set and neutrosophic ideal.

\section{Acknowledgement}

The author is highly thankful to the Referees for their valuable comments to improve the paper.

\section{References}

[1] V. Chinnadurai, K. Bharathivelan, Cubic bi-ideals in near-rings, International Journal of computer and Mathematical Sciences, 5(2)(2016), 44 - 52.

[2] V. Chinnadurai, K. Bharathivelan, Cubic Lateral Ideals in Ternary Near - Rings, International Advanced Research Jou rnal in Science, Engineering and Technology, 3(11)(2016), 209 - 215.

[3] J.S.Golan, Semirings and Their Applications, Kluwer Academic Publishers, (1999).

[4] M. Henriksen, Ideals in semirings with commutative addition, Amer. Math. Soc. Notices, 6(1958), 321.

[5] K. Iizuka, On the Jacobson radical of semiring, Tohoku Math.J., 11(2) (1959), 409-421

[6] Y.B. Jun, M.A.Öztürk, S.Z.Song, On Fuzzy h-ideals in hemiring, Inform. Sci., 162(2004), 211-226.

[7] Y.B. Jun, S.T. Jung and M.S. Kim, Cubic subgroups, An. Fuzzy Math. Inform., 2(2011), 9 - 15.

[8] Y.B. Jun, C.S. Kim and K.O. Yang, Cubic sets, An. Fuzzy Math. Inform., 4(2012), 83 - 98.

[9] A. Khan, Y.B. Jun, S.I.A. Shah, M. Ali, Characterizations of hemirings in terms of cubic h-ideals, Soft Comput., DOI 10.1007/s00500-014-1396-4.

[10] D.R.La Torre, On h-ideals and k-ideals in hemirings, Publ. Math. Debrecen, 12(1965), 219-226.

[11] X. Ma, J. Zahn, Fuzzy h-ideals in h-hemiregular and h-semisimple $\Gamma$-hemirings, Neural Comput and Applic, 19(2010), 477-485.

[12] D. Mandal, On Cubic h-ideals of $\Gamma$-hemiring, Bull. Int. Math. Virtual Inst., 10(3) (2020), 567-579.

[13] S.K. Sardar, D. Mandal, On fuzzy h-ideals in h-regular $\Gamma$-hemiring and h-duo $\Gamma$-hemiring, Gen. Math. Notes,2(1),(2011), 64-85.

[14] S.K. Sardar, D.Mandal, On fuzzy h-ideal in Г-hemiring, Int. J. Pure. Appl. Math, 56(3) (2009), 439-450.

[15] A. Rosenfeld, Fuzzy groups, J. Math. Anal. Appl., 35(1971), 512-517.

[16] L.A. Zadeh, Fuzzy Sets, Inf. Control, 8(1965), 338-353. 